\title{
DETERMINACION DE LA FRECUENCIA DE SIEMBRA DEL CULTIVO ASOCIADO CAUPI (Vigna unguiculata W.) Y MAIZ. (Zea mays L.)
}

(*) CARDAMA I. 0.

\section{RESUMEN}

Durante los años de 1982 a 1984, en el campo experimental Muyuy (suelos inundables), se estudió la frecuencia de siembra en el cultivo asociado caupí-maíz, con el fin de determinar el periodo óptimo de siembra y su implicación económica.

A nalizando las producciones del caupí y maíz como cultivos solos, formando parte del sistema, se encontró que la siembra adelantada de 15 días del caupí respecto al maíz, reportó rendimiento significativo $(885 \mathrm{Kg} / \mathrm{Ha}$.), mientras que para el maíz se logró el mejor rendimiento $(2,565 \mathrm{Kg} / \mathrm{Ha}$. $)$, cuando hubo un adelanto de 10 días de siembra sobre el caupí. En forma combinada, resultó ser más ventajosa la siembra simultánea de ambos cultivos, por su mejor aporte económico (1' 140,262 soles/Ha.)

\section{SUM M ARY}

The timing of sowing in the mixed cowpea-corn cultivation in order to determine the optimun period for sowing and its economic implication, was studied in the experimental field at M uyuy (flooded soils) between the years 1982 to 1984.

A nalizing the cowpea-corn yield as separate cultivations forming part of the sistem, it was determined that the sowing of cowpea 15 days ahead of the corn had a significant yield ( $885 \mathrm{k} . \mathrm{g} / \mathrm{Ha}$.), but the corn yielded had the best yield $(2,565 \mathrm{~kg} / \mathrm{Ha}$ ). when it was sowed 10 days ahead of cowpea in mixed sowing the simultaneons sowing of both crops proved more advantageons because of its better economic rendering (1' 140,262 soles/Ha)

PALABRAS CLAVES: Época de siembra, A sociación Caupí-M aíz.

Ingeniero Agrónomo, Investigador agrario en el cultivo de leguminosa de grano. Estación Experimental Agraria "San Roque"-Iquitos-Perú. 


\section{INTRODUCCION}

Gran parte de los productos agrícolas procedentes de la región amazónica como de otras regiones tropicales del mundo, se originan en áreas en que se practica frecuentemente una agricultura basada principalmente en cultivos asociados de diversos sembríos, durante un mismo período de tiempo; entre estos cultivos están incluidos el caupí, fríjol, maíz, yuca, plátano, etc.

L a investigación agrícola generalmente ha sido enfocada hacia el mejoramiento de variedades e híbridos y hacia el desarrollo de sistemas y tecnologías para monocultivos, dejando de lado los sistemas tradicionales de cultivos asociados (5); sin embargo, se observa que, cuando se determina el sistema óptimo de asociación para determinada región, su produce con frecuencia un aumento de producción en estos sistemas complejos (6), además de presentar como ventajas la minimización de riesgos por fluctuaciones ambientales aleatorias, utilización racional de mano de obra y balance equilibrado de la dieta alimenticia (8).

L a siembra intercalada de una leguminosa (fríjol) con un cereal (maíz), se hace para obtener un aprovechamiento del terreno; por otra parte, este tipo de asociación resulta mutuamente beneficioso, dado que las leguminosas mejoran la aereación del suelo, en tanto que los cereales proporcionan sombra, permitiendo reducir la temperatura de las leguminosa (2).

Las fechas de siembra de los cultivos asociados han sido definidos por FLOR Y FRANCIS (4), como los días transcurridos a partir de la siembra de un cultivo con respecto a otro, tratando con ello de buscar un equilibrio en el crecimiento de ambos cultivos.

Se habla de equilibrio, porque pueden haber situaciones de competencia, ya sea por factores, como luz, agua, nutrientes, así como de complementación, como en el caso del soporte que un cultivo presta a otro; el concepto fundamental es minimizar la competencia y maximizar la complementación y/o transferencia entre ellos.

En Iquitos, AGUILAR (1) y CARDAMA (3), han desarrollado estudios de cultivos asociados (caupí-maíz) en relación con distanciamientos y densidades de siembra, sirviendo como punto de partida para iniciar el presente trabajo de investigación, el que ha tratado de encontrar el período óptimo de siembra de este cultivo asociado y sus implicancias económicas.

En el plano internacional, se tienen antecedentes de trabajos similares, mas que todo sobre fechas de siembra del cultivo de fríjol con relación al maíz y yuca, 
evidenciando las ventajas de sembrar plantas alimenticias asociadas con fechas relativas de siembra, de un cultivo hacia otro.

Es así que en Colombia, FRANCIS Y TEMPLE, citados por la Universidad Nacional A graria (12) de Lima, determinaron que una ventaja de 15 días de siembra del fríjol sobre el maíz, mostró rendimientos más altos, demostrándose que con las siembras adelantadas de fríjol, según PRAGER (9), se obtiene las más altas producciones en éste y con siembras posteriores a la del maíz, los rendimientos del fríjol disminuyen significativamente.

En Brasil, PIERE DE MATOS Y LOY OLA (10), al estudiar la asociación de yuca con fríjol, encontraron que la siembra de ambos cultivos en forma simultánea, resulta como alternativa más segura de producción; además de la siembra simultánea, en otras investigaciones encontraron que también es factible sembrar con una diferencia entre las fechas de siembra de una semana. Esta práctica la verificaron en muchos experimentos THUNG Y COCK, mencionados por LEIHNER (7), asociando yuca con otras leguminosas o con maíz y ha dado resultados igualmente positivos.

\section{MATERIALES Y METODOS}

El estudio fue conducido durante los años de 1982, 1983 y 1984 en el campo experimental de Muyuy, perteneciente a la Estación Experimental Agrícola "San Roque", del CIPA XXII .L oreto, en suelos de naturaleza aluvial (Inundables periódicamente), cuyas condiciones climáticas y características físico-químicas se muestran en los Cuadros 1 y 2 , respectivamente.

Se utilizaron las variedades mejoradas Porvenir - 1 (caupí) y Cuban Y ellow (maíz), teniendo como características nueve tratamientos, conformados por la siembra del caupí y maíz en monocultivo, siembra simultánea de ambos cultivos, siembra con adelantos de 5, 10 y 15 días del maíz sobre el caupí y siembra con adelanto de 5, 10 y 15 días del caupí sobre el maíz, dispuestos en un diseño experimental de bloques completos randomizados con 4 repeticiones, teniendo como longitud cada parcela $6.00 \mathrm{~m}$. de largo $3.20 \mathrm{~m}$. de ancho, con un área neta de $11.40 \mathrm{~m}^{2}$

La siembra para el primer experimento se realizó del 25 de agosto al 10 de setiembre de 1982, para el segundo experimento del 12 al 27 de agosto de 1983 y para el tercer experimento del 23 de agosto al 8 de setiembre de 1984, de acuerdo cada tratamiento establecido, con distanciamiento de $8.80 \mathrm{~m}$. x $0.30 \mathrm{~m}$. y $0.80 \mathrm{~m} . \mathrm{x}$ $0.60 \mathrm{~m}$. de caupí y maíz, respectivamente en forma intercalada, considerando 2 plantas/mata en ambos cultivos. 
La cosecha se inició en el primer experimento con el cultivo de caupí el 29 de octubre y finalizó con el cultivo de maíz el 3 de diciembre de 1982, mientras que el segundo experimento ocurrió de I 13 de octubre (caupí) al 5 de diciembre de 1983 (maíz); finalmente, el tercer experimento se llevó a cabo del 16 de octubre:, (caupí) al 14 de diciembre de 1984 (maíz). 
CUADRO 1

DATOS CLIMATOLOGICOS PROMEDIOS DEL CAMPO EXPERIMENTAL CORRESPONDIENTE A LOS MESES DE EXPERIMENTACION

\begin{tabular}{|c|c|c|c|c|c|c|c|c|}
\hline \multicolumn{2}{|l|}{ MESES } & \multicolumn{2}{|c|}{ Temperatura en $\mathrm{C} \underline{0}$} & \multicolumn{2}{|c|}{ H umedad Relativa \% } & \multicolumn{2}{|c|}{ Precipitación } & \multirow[b]{2}{*}{ M edia } \\
\hline (m.m.) & Máxima & Mínima & M edia & Máxima & & M ínima & & \\
\hline Julio & 29.8 & 20.8 & 25.3 & 98 & 44 & & 80.5 & 181.7 \\
\hline A gosto & 30.2 & 20.7 & 25.4 & 98 & 36 & & 81.0 & 143.8 \\
\hline Setiembre & 29.7 & 20.9 & 25.3 & 92 & 42 & & 80.0 & 171.8 \\
\hline Octubre & 31.2 & 22.3 & 26.7 & 96 & 34 & & 81.5 & 144.8 \\
\hline Noviembre & 31.4 & 20.0 & 25.7 & 97 & 34 & & 81.0 & 179.6 \\
\hline Diciembre & 30.7 & 22.2 & 26.4 & 94 & 42 & & 83.5 & 203.2 \\
\hline
\end{tabular}

FUENTE: Servicio Nacional de M eteorología e Hidrología (SENA M HI)- Iquitos 
Como plagas se presentaron, al inicio del crecimiento del caupí y maíz, gusanos de tierra (Agrotis sp), ( $F$ eltia $\mathrm{sp}$ ) y grillos, controlándose eficazmente con Ravión $85 \%$ W.P. al $0.4 \%$; durante todo el proceso vegetativo del caupí se observó crisomélidos (Diabrótica sp, Ceretona sp), siendo la aplicación con Sevín 85\% PM al $0.3 \%$ efectiva y en el crecimiento del maíz el gusano cogollero (Spodotera frugiperda), fue bien controlado también con Sevín 85\% PM al 0.4\%.

En cuanto a enfermedades, solamente se apreciaron en el cultivo de caupí brotes aislados pudrición radicular (Rhizoctonia solani, Phytiun debarianum) y virosis, producida por el mosaico rugoso del caupí, por lo que se eliminaron las plantas enfermas y se efectuó el control oportuno de plagas (crisomélidos), en el caso de virosis, para evitar su diseminación

Los datos registrados en caupí y maíz fueron porcentajes de germinación, período vegetativo, peso de 100 gramos y comportamiento de plagas y enfermedades; solamente en maíz se tomaron aspecto de planta, altura de planta, peso de mazorcas y longitud de mazorcas.

Para comparación de sistemas de cultivo se empleó el $L E R=$ Land equivalente ratio (5), el cual fue obtenido a través de la siguiente ecuación:

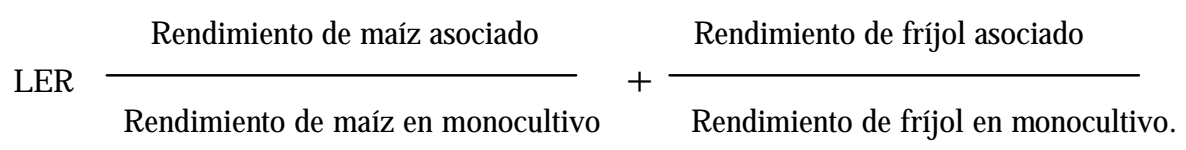

\section{RESULTADOS}

Se presentan en forma individual y combinada a través de la producción de los cultivos de caupí y maíz, conforme a las fechas de siembras programadas, bajo la modalidad de unicultivo y en asocio, así como las comparaciones de sistemas, a través del LER o UET (uso eficiente de la tierra) y sus respectivos análisis económicos; además, como un complemento de la información, se registró las observaciones fenológicas de los cultivos sujetos a estudio, durante los tres años de experimentación.

Los resultados de los experimentos efectuados por años se encuentran un los Cuadros 3 , 4 y 5.

En los Cuadros 6 y 7 se presentan resumidos los rendimientos de los tres años consecutivos de experimentación. 
CUADRO 2

ANALISIS DE CARACTERIZACION PROMEDIO DEL SUELO DONDE SE UBICARON LOS EXPERIMENTOS.

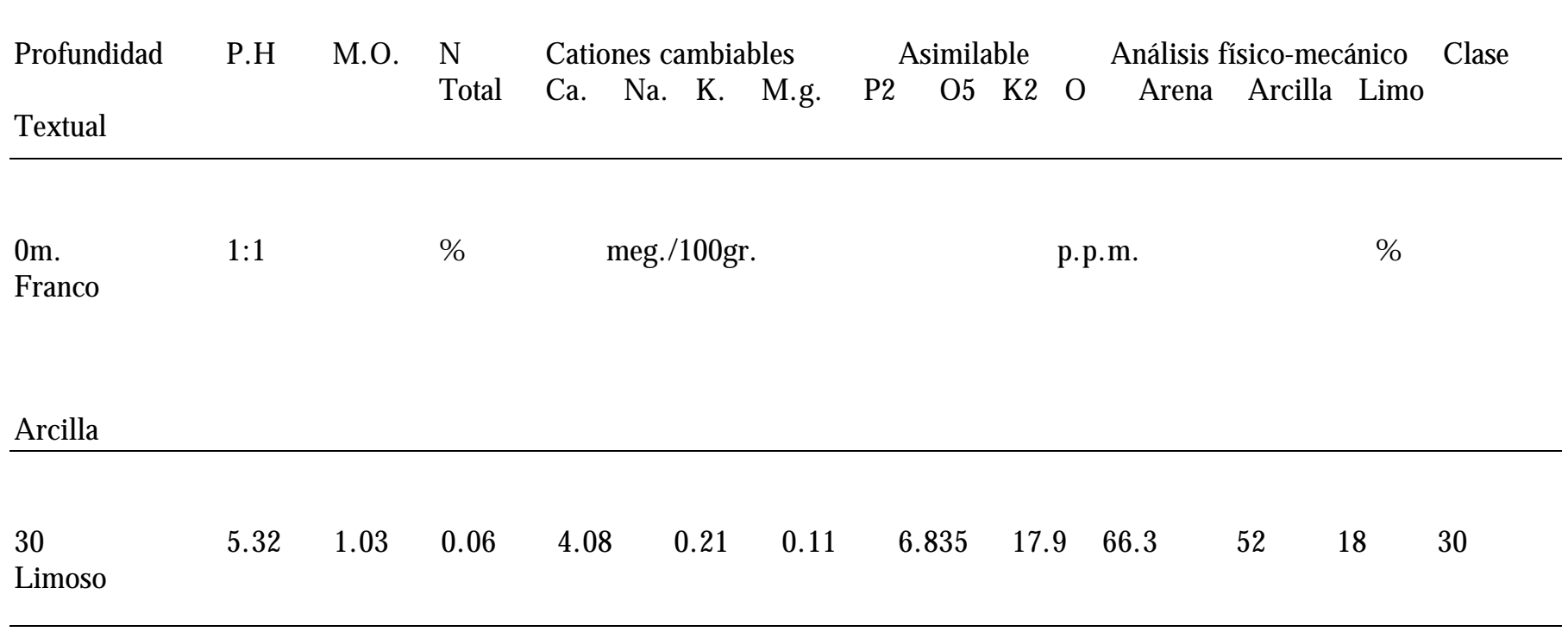




\section{CUADRO № 3}

\section{RENDIMIENTOS OBTENIDOS DEL ESTUDIO Y SU SIGNIFICANCIA ESTADISTICA \\ (Prueba de Tuckey al 5\%) \\ 1982}

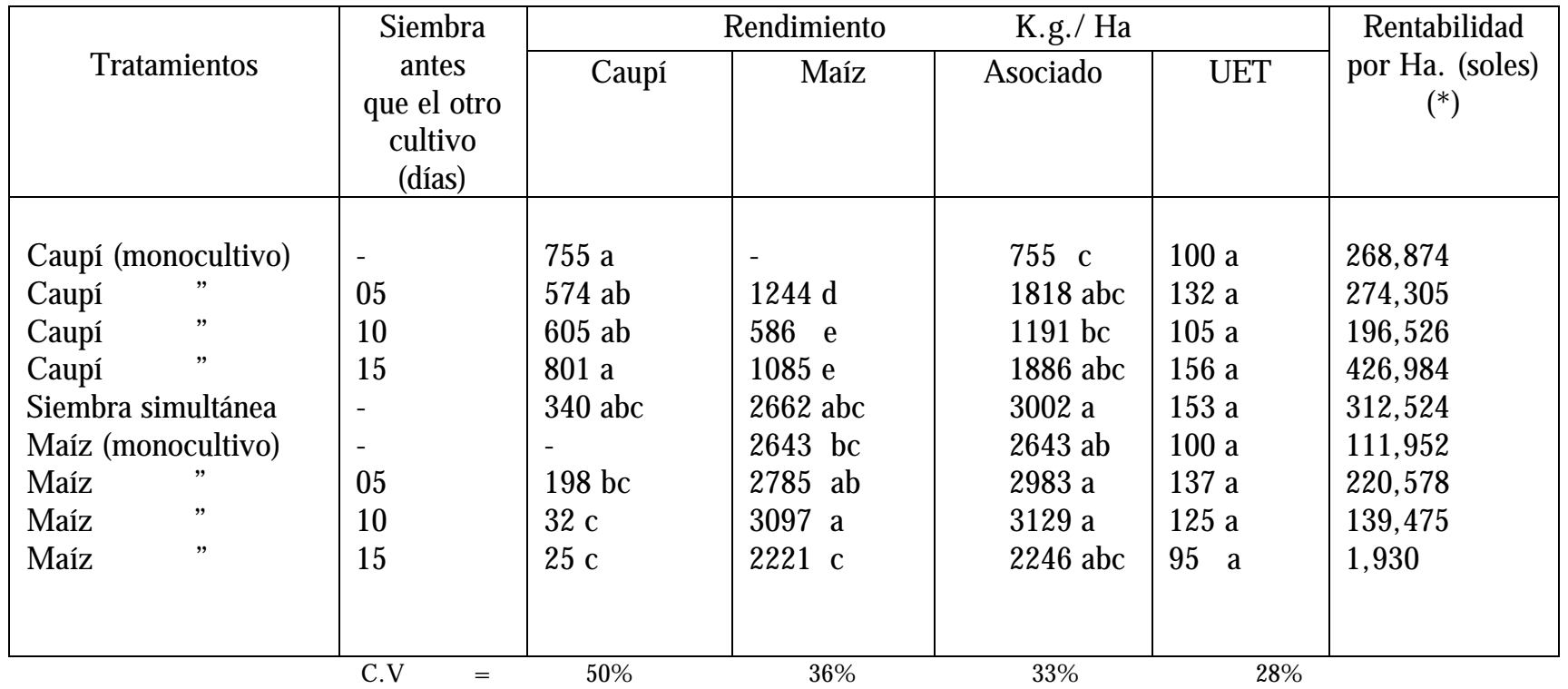

Tratamientos con letras iguales no difieren estadísticamente.

$\begin{array}{ll}\text { Observación: } & \text { Se considero para determinar la rentabilidad por Ha. Los precios de venta S/. } 800 \text { por cada K.g de caupí y de S/. } 175 \text { por cada } \\ & \text { K.g de maíz, vigentes a la fecha en el mercado de Iquitos. }\end{array}$

(*) Soles 1982 
CUADRO № 4

RENDIMIENTOS OBTENIDOS DEL ESTUDIO Y SU SIG NIFICANCIA ESTADISTICA

(Prueba de Tuckey al 5\%)

1983

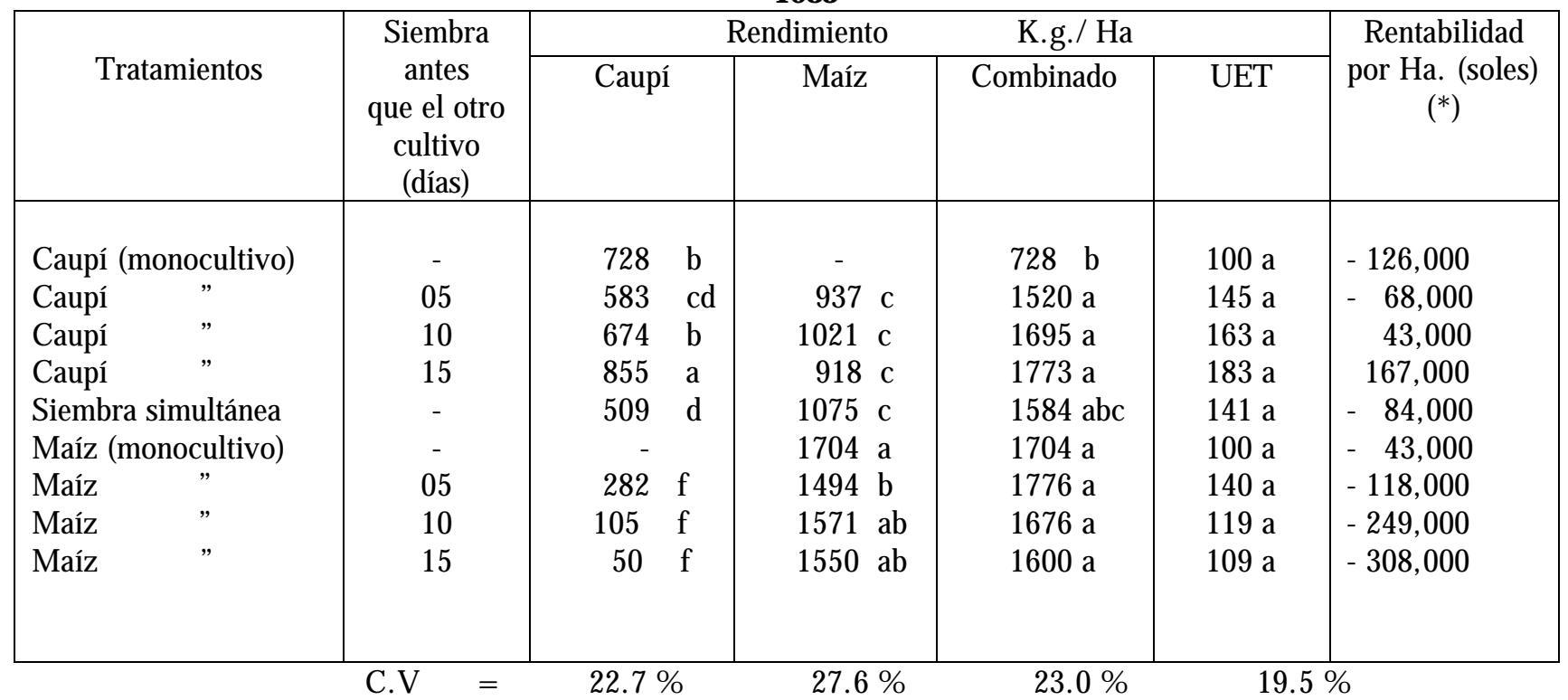

Tratamientos con letras iguales no difieren estadísticamente.

Observación:

Se considero para determinar la rentabilidad por Ha. Los precios de venta S/. 1,000 por cada K.g de caupí y de S/. 500 por cada K.g de maíz, vigentes a la fecha en el mercado de Iquitos.

(*) Soles 1983 
CUADRO No 5

RENDIMIENTOS OBTENIDOS DEL ESTUDIO Y SU SIG NIFICANCIA ESTADISTICA
(Prueba de Tuckey al 5\%)

1984

\begin{tabular}{|c|c|c|c|c|c|c|}
\hline \multirow[b]{2}{*}{ Tratamientos } & \multirow{2}{*}{$\begin{array}{c}\text { Siembra } \\
\text { antes } \\
\text { que el otro } \\
\text { cultivo } \\
\text { (días) }\end{array}$} & \multicolumn{2}{|c|}{ Rendimiento } & \multicolumn{2}{|l|}{ K.g./ Ha } & \multirow{2}{*}{$\begin{array}{c}\text { Rentabilidad } \\
\text { por Ha. (soles) } \\
(*)\end{array}$} \\
\hline & & Caupí & M aíz & Combinado & UET & \\
\hline Caupí (monocultivo) & 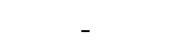 & $1285 a$ & - & $1285 \mathrm{C}$ & $100 \mathrm{a}$ & $1^{\prime} 585,000$ \\
\hline Caupí & 05 & 625 bc & $1332 b$ & 1957 bc & $95 \mathrm{a}$ & 830,000 \\
\hline Caupí & 10 & $732 \quad b c$ & $815 \mathrm{C}$ & $1547 \mathrm{C}$ & $111 \mathrm{a}$ & 734,000 \\
\hline Caupí & 15 & $998 \quad a b$ & $1263 \mathrm{c}$ & $2261 a b c$ & $122 \mathrm{a}$ & $1^{\prime} 691,000$ \\
\hline Siembra simultánea & - & 726 bc & $2836 a$ & $3562 a$ & $150 \mathrm{a}$ & $2^{\prime} 136,000$ \\
\hline M aíz (monocultivo) & _ & & $3043 a b c$ & $3043 a b$ & $100 \mathrm{a}$ & 465,000 \\
\hline M aíz " " & 05 & $490 \mathrm{~cd}$ & 2919 a & $3409 a b$ & $132 a$ & $1^{\prime} 615,000$ \\
\hline M aíz & 10 & $327 \mathrm{~cd}$ & 3026 a & $3353 a b$ & $127 a$ & $1^{\prime} 291,000$ \\
\hline M aíz & 15 & $112 \mathrm{~d}$ & $2430 a$ & $2542 a b c$ & $90 a$ & 339,000 \\
\hline
\end{tabular}

Tratamientos con letras iguales no difieren estadísticamente.

Observación: Se considero para determinar la rentabilidad por Ha. Los precios de venta S/. 2,500 por cada K.g de (*) Soles 1984 caupí y de S/. 750 por cada K.g de maíz, vigentes a la fecha en el mercado de Iquitos. 
CUADRO № 6

RENDIMIENTOS OBTENIDOS DE L ESTUDIO Y SU SIG NIFICANCIA ESTADISTICA

(Prueba de Tuckey al 5\%)

\begin{tabular}{|c|c|c|c|c|c|c|}
\hline \multirow[b]{2}{*}{ Tratamientos } & \multirow{2}{*}{$\begin{array}{c}\text { Siembra antes } \\
\text { que el otro } \\
\text { cultivo (días) }\end{array}$} & \multicolumn{2}{|c|}{ Rendimiento } & \multicolumn{2}{|l|}{$\mathrm{K} . \mathrm{g} / \mathrm{Ha}$} & \multirow{2}{*}{$\begin{array}{c}\text { Rentabilidad } \\
\text { por } \mathrm{Ha} \text {. (soles) } \\
(*)\end{array}$} \\
\hline & & Caupí & M aíz & A sociado & UET & \\
\hline Caupí & - & 923 a & - & $923 d$ & $100 a$ & 800,937 \\
\hline (monocultivo) & 05 & $594 \quad c$ & $1171 \mathrm{~b}$ & $1765 b c$ & $124 \mathrm{a}$ & 484,152 \\
\hline Caupí & 10 & 670 bc & $807 \mathrm{~b}$ & 1477 c & $126 \mathrm{a}$ & 324,509 \\
\hline Caupí & 15 & $885 a b$ & $1089 \mathrm{~b}$ & $1974 a b c$ & $154 \mathrm{a}$ & 761,661 \\
\hline Caupí & - & $525 \mathrm{~cd}$ & $2191 \mathrm{a}$ & $2716 a$ & $148 \mathrm{a}$ & $1^{\prime} 140,262$ \\
\hline Siembra simultánea & - & - & $2463 a$ & $2463 a b$ & $100 \mathrm{a}$ & 245,476 \\
\hline M aíz (monocultivo) & 05 & 323 de & $2399 a$ & $2722 \mathrm{a}$ & $136 a$ & 799,789 \\
\hline M aíz & 10 & $155 \mathrm{e}$ & $2565 a$ & $2720 a$ & $124 \mathrm{a}$ & 466,237 \\
\hline M aíz & 15 & $62 \mathrm{e}$ & $2067 a$ & $2129 a b c$ & $98 \mathrm{a}$ & - 137,535 \\
\hline M aíz & & & & & & \\
\hline
\end{tabular}

Tratamientos con letras iguales no difieren estadísticamente.

(*) Soles 1984 


\section{CUADRO 7}

\section{OBSERVACIONES FENOLOGICAS PROMEDIOS REGISTRADOS SEGUN} TRATAMIENTOS DE LOS CULTIVOS SUJETOS A ESTUDIOS

Tratamientos Cultivos InicioPeriodoAspectoAlturaPeso Long.Peso ConmGermi-flora-vegeta- planta Plantamazor-mazor- (gr.) pt. nación ción tivo

(m.) ca ca (cm.)100 plagas (dias) (dias) (kg.) granos enfdes.

\begin{tabular}{|c|c|c|c|c|c|c|c|c|c|c|}
\hline Caupi (monocultivo) & caupi & 89 & 39 & 67 & - & - & - & - & 18 & $\mathbf{T}$ \\
\hline \multirow{2}{*}{$\begin{array}{l}\text { caupi sembrado } 5 \text { dias } \\
\text { antes del } \\
\text { maiz }\end{array}$} & caupí & 90 & 39 & 67 & - & - & - & - & 18 & $T$ \\
\hline & maiz & 82 & 53 & 105 & regular & 2.31 & 0.727 & 19 & 325 & $\mathbf{R}$ \\
\hline \multirow{2}{*}{$\begin{array}{l}\text { Caupí sembrado } 10 \\
\text { días antes del } \\
\text { maiz }\end{array}$} & caupí & 88 & 39 & 67 & - & - & - & - & 16 & $\mathrm{~T}$ \\
\hline & maíz & 85 & 53 & 104 & regular & 2.21 & 0.571 & 18 & 321 & $\mathbf{R}$ \\
\hline \multirow{2}{*}{$\begin{array}{l}\text { Caupi sembrado } 15 \\
\text { dias antes del } \\
\text { maiz }\end{array}$} & caupi & 91 & 39 & 67 & - & - & - & - & 21 & $\mathrm{~T}$ \\
\hline & maiz & 82 & 53 & 101 & regular & 2.25 & 0.582 & 20 & 324 & $\mathrm{R}$ \\
\hline \multirow[t]{2}{*}{ Siembra simultánea } & caupí & 89 & 39 & 67 & - & - & - & - & $16-$ & $\mathrm{T}$ \\
\hline & Imaiz & 83 & 53 & 107 & regular & 2.27 & 0.755 & 18 & 327 & $\mathrm{R}$ \\
\hline Maiz (mono & maiz & 87 & - & 108 & regular & 2.33 & 0.89 & 20 & 334 & $\mathrm{R}$ \\
\hline \multirow{2}{*}{$\begin{array}{l}\text { Maiz sembrado } 5 \text { dias } \\
\text { antes del } \\
\text { caupi }\end{array}$} & caupi & 90 & 40 & 68 & - & - & - & - & 18 & $\mathrm{~T}$ \\
\hline & maiz & 87 & 53 & 108 & regular & 2.341 & 0.746 & 19 & 333 & $\mathrm{R}$ \\
\hline \multirow{2}{*}{$\begin{array}{l}\text { Maiz sembrado } 10 \\
\text { días antes del } \\
\text { caupi }\end{array}$} & c & 91 & 40 & 9 & - & - & - & - & 16 & $\mathrm{~T}$ \\
\hline & maíz & 87 & 53 & 110 & regular & 2.49 & 0.761 & 20 & 331 & $\mathrm{R}$ \\
\hline \multirow{2}{*}{$\begin{array}{l}\text { Maíz sembrado } 15 \\
\text { dias antes del } \\
\text { caupi }\end{array}$} & caup & 86 & 40 & 70 & - & - & - & - & 15 & $\mathrm{~T}$ \\
\hline & maíz & 83 & 53 & 112 & regular & 2.46 & 0.695 & 20 & 330 & $\mathbf{R}$ \\
\hline
\end{tabular}




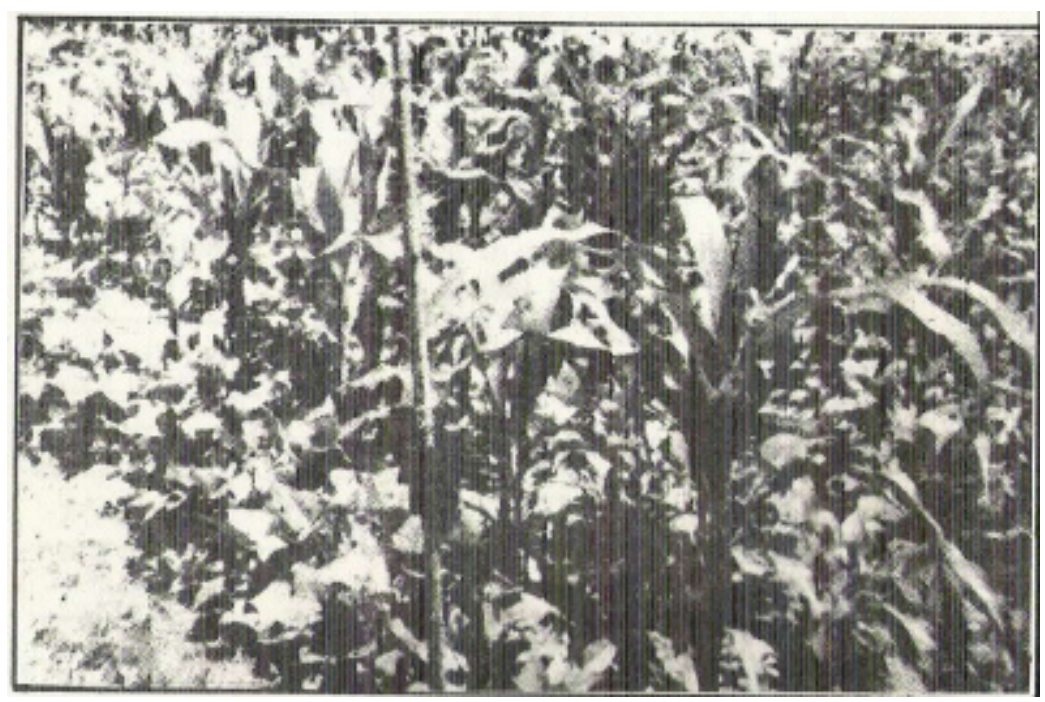

\section{FOTO 1}

Parcelas sembradas con los cultivos asociados caupí maíz e forma simultanea en inicio de desarrollo vegetativo 


\section{DISCUSION}

El trabajo efectuado durante los años de experimentación estuvo relacionado a una misma zona agroecológica, caracterizada por tener suelos de origen aluvial, siendo efectuada en un área de restinga baja, lugar representativo de la producción agrícola de la región, cuyos resultados reportados en los Cuadros 3 al 7, muestran que existe una influencia de las fechas relativas de siembra de los cultivos asociados caupí-maíz.

En 1982 (Cuadro 3), se encontró que los mayores rendimientos de grano seco del caupí como monocultivo, se obtuvieron cuando se adelantó la siembra en 15 días al maíz (801 $\mathrm{Kg} / \mathrm{Ha}$.), mientras que retrasando la siembra del caupí en 15 días con respecto al maíz, los rendimientos bajaron significativamente a $25 \mathrm{Kg} / \mathrm{Ha}$.; del mismo modo, el mayor rendimiento de maíz como monocultivo y en forma asociada ocurrió cuando el maíz fue sembrado 10 días antes del caupí. Sin embargo, la relación de equivalencia de terreno (LER) y la rentabilidad fue más alta cuando el caupí fue sembrado 15 días antes del maíz.

En el segundo año de experimentación (1983), nuevamente cuando el caupí fue sembrado con 15 días de adelanto sobre maíz, los rendimientos corno monocultivo fueron mayores $(855 \mathrm{Kg} / \mathrm{Ha}$ )); por el contrario, cuando la siembra del caupí se retrasé en 15 días respecto al maíz, la producción se vio ostensiblemente afectada (50 $\mathrm{Kg} / \mathrm{Ha}$.), en tanto que la siembra del maíz corno monocultivo dio el mayor rendimiento (91 $\mathrm{Kg} / \mathrm{Ha}$ ), cuando el maíz fue adelantado por la siembra del caupí en 15 días.

Sin embargo, analizando el rendimiento en forma combinada, rindió más cuando el maíz fue sembrado con 5 días de anticipación sobre el caupí y solamente fue significativo con la siembra del caupí en monocultivo, pero la relación equivalente del terreno y la rentabilidad fue superior con la siembra adelantada del caupí en 15 días sobre el maíz.

En el último año de experimentación (1984), la siembra del caupí sólo como unicultivo y con un adelanto de 15 días sobre el maíz, fueron los sistemas de siembra que dieron los mejores rendimientos significativos, al igual que la siembra del maíz sólo y con un adelanto de 10 días sobre el caupí, los cuales fueron significativos a la siembras retrasadas de 5,10 y 15 días con respecto al caupí; no obstante a esto, con la siembra simultánea de ambos cultivos, fue mayor la producción como asocio, así como el índice del uso eficiente de tierra y la rentabilidad. 
En el Cuadro 6, en forma resumida, se presentan los rendimientos promedios de los 3 años de experimentación, con sus respectivos análisis estadísticos combinados, observándose que el rendimiento de grano seco del cultivo de caupí, así como del maíz, se han visto afectados con la siembra adelantada de uno hacia otro cultivo.

En el caupí asociado con maíz con un adelanto de 15 días de siembra, el rendimiento se ha visto favorecido, mientras que siembras atrasadas influyeron negativamente en su producción, decreciendo hasta $62 \mathrm{Kg} / \mathrm{Ha}$., cuando se retraso en 15 días de siembra respecto al maíz, concordando con los resultados encontrados por FRANCIS, FLOR Y TEMPLE en el CIAT-COLOMBIA, mencionado por la UNA (12), donde una ventaja de 15 días en la siembra del fríjol mostró los rendimientos más altos, lo cual fue confirmado por arios pequeños agricultores de zonas aledañas que practican este sistema; de igual forma sucedió en el cultivo de maíz, donde una ventaja de 10 días de siembra respecto al caupí produjo el mayor rendimiento y retrasando sus siembras disminuyeron significativamente sus producciones, confirmando los resultados encontrados por PRAGER en el CIAT-COLOM BIA (9), al asociar fríjol con maíz en diferentes fechas relativas de siembra.

Combinando los rendimientos, destacan las siembras de maíz con 5 y 10 días de adelanto sobre el caupí y la forma simultánea de ambos cultivos; éste último con mayor índice de equivalencia de terreno, después de la siembra del caupí con un adelanto de 15 días sobre el maíz y con una mayor ventaja económica al actuar como siembra simultánea, resultando de esta forma como la mejor alternativa de siembra por el mayor reporte de utilidad, tal como hace mención TEJADA y et al (11)

Conforme a las observaciones fenológicas (Cuadro 7) promedios registrados, se nota que los cultivos sujetos al sistema de estudio presentaron buena germinación; el inicio de floración ocurrió casi en el mismo tiempo, conforme al cultivo, solamente existió un día de diferencia en el caupí, cuando fue alternado después de la siembra del maíz, sucediendo igual con su período vegetativo.

En el caso del maíz, cuando este fue sembrado con días de adelanto sobre el caupí, su período vegetativo se prolongó, también se experimentó variaciones de otras características fenológicas, probablemente estas fluctuaciones fueron debidas a la competencia entre los cultivos por mayor o menor luminosidad, cuando fueron sembradas con adelantos o retrasos de un cultivo sobre el otro. 


\section{REFERENCIAS BIBLIOGRAFICAS}

AGUILAR, R. M. 1976. Cultivo asociado maíz (Zea mays), fríjol chiclayo (Vigna sinensis) en la zona de Iquitos. Tesis Ingํ. A grónomo, Programa A gronomía UNAP. Iquitos-Perú. 52p.

CIAT (1987). La fijación de nitrógeno en el fríjol Noti-CIAT, serie A S-G. CIATColombia.

CARDAMA, V. 1. 0. (1984). Cultivos asociados caupí-maíz. A vances de Investigación, vol. № 1, A gosto. INIPA -CIPA XXII. Iquitos-Perú $8 \mathrm{p}$.

FLOR, C.A. y C. A. FRANCIS (1975). Propuesta de estudio de algunos componentes de una metodología para investigar los cultivos asociados en trópico latinoamericano. XXII Reunión de PCCM CA. San Salvador, Salvador. $37 p$.

NCIS, C. A. (1978). Fríjol/maíz: Principios de la asociación y prioridades de la investigación. CIAT-Cali, Colombia. 11p.

FRANCIS, C. A. FLOR y M. PRAGER (1987). Contrastes agronómicos entre el monocultivo de maíz y la asociación maíz-fríjol. CIAT-Cali, colombia. 23 p.

LEIHNER, D.(1983). Y uca en cultivos asociados. Manejo y evaluación. Centro Internacional de Agricultura Tropical. CIA T-Cali, Colombia. 79 p.

OKIGBO, B. N.Y. D. J. GRENLAND (1976). Intercropping systems in tropical A frica. ASA Special publ. № 27, pp. 63-101.

PRAGER, M. (1977). Factores agronómicos en la producción fríjol/maíz. Información de resultados de investigación. CIAT- Palmira. Colombia. 17 p.

PIERE DE MATTOS P. L. Y. D. J. L. LOY OLA (1981). U tilizao de cultivo de mandioca consorciada con frijao. EM BRA PA-Centro Nacional de Pesquiza de mandioca e fruticultura. Circular Técnica $\mathrm{N} N 0$ 02. Cruzadas Almas-Bahía, Brasil, $22 \mathrm{p}$.

TEJADA, G. (1981). Factores agronómicos en la asociación fríjol-maíz. Curso intensivo de adiestramiento, Post-Grado en investigación para la producción de frijol en el Perú. Lima-Perú. 18 p.

UNIVERSIDAD NACIONAL AGRARIA 1976. Informativo de maíz. Programa Cooperativo de Investigación en Maíz. Boletín № 14. Lima-Perú. 